\title{
Qualitative and Quantitative Performance Evaluation of Adhoc on Demannd Routing Protocol in Manet
}

\author{
Patil V.P. \\ Smt. Indira Gandhi College of Engineering, New Mumbai, India
}

\begin{abstract}
A mobile ad hoc network (MANET) is a network consisting of a set of wireless mobile nodes that communicate with each other without centralized control or established infrastructure. Every node in MANET moves arbitrarily making the multi-hop network topology to change randomly at unpredictable times. There are several familiar routing protocols like reactive, proactive, hybrid routing protocols which have been proposed for providing communication among all the nodes in the network. This paper presents Qualitative and Quantitative Analysis of Reactive Routing Protocol Performance Evaluation in Mobile Ad Hoc Network for protocols such as DYMO, LAR, ABR, TORA, AODV and DSR based on metrics such as security, multicasting, scalability, Packet delivery ratio and average end-to-end delay, Routing load and network life time by using the NS-2 simulator. The results shows that there is no routing protocol in the current stage without modifications that can provide efficient routing to any size of network, regardless of the number of nodes and the network load and mobility. AODV shows better performance than TORA and DSR.
\end{abstract}

Index Terms: AODV, TORA, ABR, DSR, Routing protocols, NS-2.

\section{Introduction}

A mobile ad hoc network (MANET) is a network comprising wireless mobile nodes (MNs) that communicate with each other without centralized control or established infrastructure [1]. One of the distinctive features of MANET is, each node must be able to act as a router to find out the optimal path to forward a packet. As nodes may be mobile, entering and leaving the network, the topology of the network will change continuously. MANETs provide an emerging technology for civilian and military applications. Since the medium of the communication is wireless, only limited bandwidth is available. Another important constraint is energy due to the mobility of the nodes in nature.

Routing protocols are divided into two categories: Proactive and Reactive. Proactive routing protocols are table-driven protocols and they always maintain current up-to-date routing information by sending control messages periodically between the hosts which update their routing tables. The proactive routing protocols use link-state routing algorithms which frequently flood the link information about its neighbors [2.] Reactive or ondemand routing protocols create routes when they are needed by the source host and these routes are maintained while they are needed. Such protocols use distance-vector routing algorithms [3].

This paper provides an analysis and performance evaluation of different reactive routing protocols that may be suitable for wireless communications. The evaluation is conducted in two phases. In the first phase, comparison of the protocols based on qualitative metrics is done to locate those that may fit the evaluation criteria. In the second phase, evaluation of the selected protocols is done from the first phase based on quantitative metrics in a mobility scenario and node density. Therefore, the objectives are: i) to compare them and select those protocols based on qualitative metrics that most satisfy the IETF design considerations; ii) to evaluate the selected protocols through extensive simulations under the same network parameters and mobility scenario. The rest of this paper is organized as follows. The next section provides an overview of the related work in the area of evaluation of routing protocols for wireless ad hoc networks. Routing protocols for MANET are discussed in Section 3.The protocols under considerations are discussed in section 4. In Section 5, the performance metrics is described under which evaluation has been done. In Section 6, three routing protocols based on qualitative metrics are evaluated. Results from extensive ns-2 simulations based on quantitative metrics are presented in Section 7. Section 8 concludes this paper.

\section{Related Work}

A number of routing protocols have been proposed and implemented for MANETs in order to enhance the bandwidth utilization, higher throughputs, lesser overheads per packet, minimum consumption of energy and others. All these protocols have their own advantages and disadvantages under certain circumstances. MANET Routing Protocols possess two properties such as Qualitative properties (distributed operation, loop freedom, demand based routing \& security) and Quantitative properties (end-to-end throughput, delay, route discovery time, memory byte requirement \& network recovery time). Obviously, most of the routing protocols are 
qualitatively enabled. A lot of simulation studies were carried out in the paper [4] to review the quantitative properties of routing protocols.

Performance comparison of AODV and DSR routing protocols in a constrained situation is done in [5]. The authors claim that the AODV outperforms DSR in normal situation but in the constrained situation DSR out performs AODV, where the degradation is as severe as 30\% in AODV whereas DSR degrades marginally as $10 \%$.

Ahmed and Alam [6] compare three routing protocols (DSR, AODV, and TORA) through simulations conducted with a discrete-event simulator (OPNET Modeler 10.5 version).Simulation results indicate that under specific simulation parameters TORA presents a higher performance than AODV and DSR.

Perkins et all [7] show the performance of two on demand routing protocols namely DSR and AODV. Though both AODV and DSR use on demand route discovery, they have different routing mechanics. The authors observe that for application oriented metrics such as delay, throughput DSR outperforms AODV when the numbers of nodes are smaller. AODV outperforms DSR when the number of nodes is very large. The authors do show that DSR consistently generate less routing load than AODV.

In Kumar et al. [8], a comparison of the performance of two prominent on-demand reactive routing protocols for MANET (DSR and AODV) is presented, along with the traditional proactive DSDV protocol.

In Rahman and Zukarnain [9] the performance comparison between three routing protocols, namely AODV, DSDV and an improvement of DSDV, is presented. The authors use three network metrics, namely packet delivery ration, end-to-end delay, and routing overhead.

In Divecha et al. [10] the effects of various mobility models on the performance of DSR and AODV are studied. For experimental purposes, four mobility scenarios are presented: Random Waypoint, Group Mobility, Freeway and Manhattan models. Performance comparison has also been conducted across varying node densities and number of hops. The experimental results illustrate that the performance of routing protocols varies across different mobility models, node densities and length of data paths.

In [25] the different performance evaluation parameters of DYMO reactive routing protocol are discussed.

In [11] four different routing protocols like AODV, TORA, DSDV and DSR are compared. It is shown through simulation results that DSR generates less routing load than AODV. AODV suffers from end to end delay while TORA has very high routing overhead. The better performance of DSR is because it exploits caching aggressively and maintains multiple routes to the destinations.

A comparison of Link State, AODV and DSR protocols for two different traffic classes, in a selected environment is done in [12].It is claimed that AODV and DSR perform well when the network load is moderate and if the traffic load is heavy then simple Link State outperforms the reactive protocols .

This paper evaluates quantitatively in detail three reactive MANET protocols (AODV, DSR and TORA) based on four evaluation parameters (packet delivery ratio, end to end delay, routing load, network life time) by varying pause time and node density with focus on the Random way point Mobility (RWP) model.

\section{Mobile Ad Hoc Network Routing Protocols}

There are many ways to classify the MANET routing protocols, depending on how the protocols handle the packet to deliver from source to destination. But Routing protocols are broadly classified into three types such as Proactive, Reactive and Hybrid protocols [13].

\subsection{Proactive routing protocols}

In this category, every node in the network has one or more routes to any possible destination in its routing table at any given time. Data received from the upper transport layer are immediately transmitted, as at least one route to the destination is already in the node's routing table. Proactive protocols presents low latency but medium to high routing overhead. This is due to the nodes periodically exchange control messages and routing table information in order to keep up-to-date routes to any active node in the network. However, a node, wasting process recourses and bandwidth, may never use some of these routes. Proactive protocols can better address security vulnerabilities, because of the periodic exchange of control messages and routing table information. Thus a loss or modification of any route update can be overcome by the next scheduled update. In this category, we evaluate two proactive protocols, named Optimized Link State Routing (OLSR) [14], Destination Sequenced Distance Vector (DSDV) [15].

\subsection{Reactive Protocols}

These types of protocols are also called as On Demand Routing Protocols where the routes are not predefined for routing. A Source node calls for the route discovery phase to determine a new route whenever a transmission is needed. This route discovery mechanism is based on flooding algorithm which employs on the technique that a node just broadcasts the packet to all of its neighbors and intermediate nodes just forward that packet to their neighbors. This is a repetitive technique until it reaches the destination. Reactive techniques have smaller routing overheads but higher latency. Example Protocols: DSR, AODV, DSR, TORA, LAR, DYMO. 


\subsection{Hybrid Protocols}

Hybrid protocols are the combinations of reactive and proactive protocols and takes advantages of these two protocols and as a result, routes are found quickly in the routing zone. Example Protocol: ZRP (Zone Routing Protocol), GPSR (Greedy perimeter stateless routing).

\section{Description Of Reactive Routing Protocols Under Consideration. 4.1 Ad hoc On Demand Distance Vector Routing(AODV) Protocol}

As AODV protocol is a flat routing protocol it does not need any central administrative system to handle the routing process. AODV tends to reduce the control traffic messages overhead at the cost of increased latency in finding new routes. The AODV has great advantage in having less overhead over simple protocols which need to keep the entire route from the source host to the destination host in their messages. The RREQ and RREP messages, which are responsible for the route discovery, do not increase significantly the overhead from these control messages. AODV reacts relatively quickly to the topological changes in the network and updating only the hosts that may be affected by the change, using the RRER message. The Hello messages, which are responsible for the route maintenance, are also limited so that they do not create unnecessary overhead in the network. The AODV protocol is a loop free and avoids the counting to infinity problem, which were typical to the classical distance vector routing protocols, by the usage of the sequence numbers. [19].

\subsection{Dynamic Source Routing (DSR) Protocol}

The Dynamic Source Routing (DSR) is a reactive unicast routing protocol that utilizes source routing algorithm [17]. In DSR, each node uses cache technology to maintain route information of all the nodes. There are two major phases in DSR such as: i) Route discovery ii) Route maintenance. When a source node wants to send a packet, it first consults its route cache [18]. If the required route is available, the source node sends the packet along the path. Otherwise, the source node initiates a route discovery process by broadcasting route request packets. Receiving a route request packet, a node checks its route cache. If the node doesn't have routing information for the requested destination, it appends its own address to the route record field of the route request packet. Then, the request packet is forwarded to its neighbors. If the route request packet reaches the destination or an intermediate node has routing information to the destination, a route reply packet is generated. When the route reply packet is generated by the destination, it comprises addresses of nodes that have been traversed by the route request packet. Otherwise, the route reply packet comprises the addresses of nodes the route request packet has traversed concatenated with the route in the intermediate node's route cache. The advantage of this protocol is reduction of route discovery control overheads with the use of route cache and the disadvantage is the increasing size of packet header with route length due to source routing.

\subsection{The Temporally Ordered Routing Algorithm (TORA)}

The Temporally Ordered Routing Algorithm (TORA) [26] is a highly adaptive, efficient and scalable distributed routing algorithm based on the concept of link reversal. TORA is proposed for highly dynamic, mobile, multi hop wireless networks. It is a source initiated routing protocol. It finds multiple routes from a source node to a destination node. The main feature of TORA is that the control messages are localized to a very small set of nodes near the occurrence of a topological change. To achieve this, the nodes maintain routing information about adjacent nodes. The protocol has three basic functions: Route creation, Route maintenance and Route erasure. TORA can suffer from unbounded worst-case convergence time for very stressful scenarios. TORA has a unique feature of maintaining multiple routes to the destination so that topological changes do not require any reaction at all. The protocol reacts only when all routes to the destination are lost. In the event of network partitions the protocol is able to detect the partition and erase all invalid routes [26].

\subsection{Location Aided Routing (LAR)}

LAR [27] is based on flooding algorithms (such as DSR). However, LAR attempts to reduce the routing overheads present in the traditional flooding algorithm by using location information. This protocol assumes that each node knows its location through a GPS. Two different LAR scheme were proposed in [28], the first scheme calculates a request zone which defines a boundary where the route request packets can travel to reach the required destination. The second method stores the coordinates of the destination in the route request packets. These packets can only travel in the direction was the relative distance to the destination becomes smaller as they travel from one hop to another. Both methods limit the control over-head transmitted through the network and hence conserve bandwidth. They will also determine the shortest path (in most cases) to the destination, since the route request packets travel away from the source and towards the destination. The disadvantage of this protocol is that each node is required to carry a GPS. Another disadvantage is (especially for the first method), that protocols may behave similar to flooding protocols (e.g. DSR and AODV) in highly mobile networks. 
The Dynamic MANET On-demand (DYMO) [25] routing protocol is a simple and fast routing protocol for multihop networks. It discovers unicast routes among DYMO routers within the network in an on-demand fashion, offering improved convergence in dynamic topologies. To ensure the correctness of this protocol, digital signatures and hash chains are used. The basic operations of the DYMO protocol are route discovery and route management.

\subsection{Associativity Based Routing (ABR)}

In associativity-based routing ABR [29], protocol uses a different metrics than shortest path. It also uses the same mechanism as DSR which is aggregating the node IDs along the path to the final destination. The objective is to select a longer lived route which will help in reducing the cost of reconstructing routes. The metric used instead of the shortest hop count is the Location Stability or the Associativity between nodes. Moving nodes tend to break the associativity with their neighbors and hence they are not good Candidates to carry routes. Nodes periodically broadcast beacons to signify their existence with their neighbors. Location Stability is determined by counting the periodic beacons that a node receives from its neighbors. Links between nodes are classified into Stable and Unstable links based on the count of beacons. Source Node broadcast Route Request packets. Each neighbor will check if it received this request before or if its ID is in the list. If yes it will drop the packet. If not it will append its ID and the status of the link weather it is stable or not to the packet and rebroadcast the packet again.

\section{Performance Evaluation Metrics}

The Internet Engineering Task Force MANET working group suggests two different types of metrics for evaluating the performance of routing protocols for MANETs in RFC 2501 [22]. In accordance with RFC 2501 , routing protocols should be evaluated in terms of both qualitative metrics and quantitative metrics. In the phase, the routing protocols are located that may be suitable in high speed wireless communications based on qualitative metrics. In the second phase, the selected protocols are evaluated from the first phase based on quantitative metrics.

\subsection{Qualitative metrics}

These metrics include:

i) Security: The wireless environments, along with the nature of the routing protocols in MANETs, which require each node to participate actively in the routing process, introduce many security vulnerabilities. Therefore routing protocols should efficiently support security mechanisms to address these vulnerabilities.

ii) Loop freedom: This refers mainly, but not only, to all protocols that calculate routing information based on the Bellman-Ford algorithm. In a wireless environment with limited bandwidth, interference from neighboring nodes' transmissions and a high probability of packet collisions, it is essential to prevent a packet from 'looping' in the network and thus consuming both processing time and bandwidth.

iii) Sleep mode: In general, nodes in a MANET use batteries for their energy source. The protocol should be able to operate, even though some nodes are in 'sleep mode' for short periods, without any adverse consequences in the protocol's performance.

iv) Unidirectional link support: Nodes in the wireless environment may be able to communicate only through unidirectional links. It is preferable that routing protocols are able to support both unidirectional and bidirectional links.

v) Multicasting: Multicasting support is important especially for the transmission of real-time data (for example, multimedia data) in many nodes at the same time.

vi) On-demand routing behavior: Owing to bandwidth limitations in the wireless network, on-demand, or reactive-based, routing minimizes the dissemination of control packets in the network, increases the available bandwidth for user data, and conserves the energy resources of the mobile nodes. Reactive routing protocols introduce a medium to high latency.

vii) Distributed environment: The way of interconnecting nodes under distributed environment

viii) Periodic broadcast: It represents sending packets periodically.

ix) Scalability: The up gradation of no. of nodes in the given environment.

$\mathrm{x})$ Routing scheme: It indicates the scheme of routing like flat routing.

xi) Routing metric: It provides the path of connecting nodes for sending packets.

xii) Route optimization. The route has to be optimized for qualitative performance of sending packets.

Therefore, a routing protocol for MANETs should keep a balance between latency and routing overhead, energy consumption, and node participation in the routing process, and should employ security Mechanisms. For high speed wireless communications, low latency and high packet delivery ratio are more important than low routing overhead. Energy consumption is not always a high issue in vehicular 
communications, as nodes may well be suited in vehicular platforms with adequate energy resources. However, for portable radio devices, energy consumption is an important issue.

\subsection{Quantitative metrics}

Quantitative metrics include:

i) Route acquisition time: it indicates how much time does a protocol need to discover a route? This is a main concern in reactive routing protocols, as the longer the time is, the higher the latency is in the network.

ii) Out-of-order delivery: The percentage of packets that are delivered out of order may affect the performance of higher-layer protocols such as TCP, which prefers in-order data delivery of packets.

iii) Efficiency. Additional metrics can be used to measure the efficiency of the protocol. One can use them to measure the portion of the available bandwidth that is used by the protocol for route discovery and maintenance. Another measurement calculates the packet delivery ratio over the total number of packets transmitted and the energy consumption of the protocol for performing its task.

iv) End-to-end data throughput and delay: these metrics can be used to measure the effectiveness of the routing protocol. Design considerations that increase delay and minimize data throughput can be revealed by these metrics.

All the above quantitative metrics should be based on the same network attributes, such as network density, mobility, data density, bandwidth, energy resources, transmission and receiving power, antenna types, etc. In this paper for performance evaluation, the general ideas described in RFC 2501 is followed , and use four quantitative metrics similar to those in Das et al. [16]. The packet delivery ratio and average end-to-end delay are more important for best-effort traffic. The normalized routing load will be used to evaluate the efficiency of the routing protocol. All these metrics are defined as follows:

Packet Delivery Ratio: This is the number of packets sent from the source to the number of received at the destination.

Average end-to-end delay: This is the average time delay for data packets from the source node to the destination node.

Normalized routing load: The normalized routing load is defined as the fraction of all routing control packets sent by all nodes over the number of received data packets at the destination nodes. This metric discloses how efficient the routing protocol is. Proactive protocols are expected to have a higher normalized routing load than reactive ones. The larger this fraction is, the less efficient the protocol is.

Network Life Time: Network life time is the time when a node finished its own battery for the first time. The performance is better when network life time is high.

\section{Performance Evaluation Based On Qualitative Metrics}

In this section the evaluation results based on qualitative metrics is presented. The evaluation process requires studying the protocols and finding their attributes that satisfy the evaluation criteria of RFC 2501. This initial selection of protocols for further evaluation depends on their performance here. Table 1 shows the qualitative performance comparison.

TABLE 1: The Qualitative Performance Comparison

\begin{tabular}{|c|c|c|c|c|c|c|c|}
\hline \multirow{2}{*}{$\begin{array}{l}\text { Sr.N } \\
\text { o. }\end{array}$} & \multirow{2}{*}{$\begin{array}{l}\text { Qualitative } \\
\text { metrics }\end{array}$} & \multicolumn{6}{|c|}{ Reactive protocols } \\
\hline & & ABR & DYMO & AODV & DSR & LAR & TORA \\
\hline 1 & Security & Yes & Yes & No & No & No & Yes \\
\hline 2 & Loop freedom & Yes & Yes & Yes & Yes & Yes & Yes \\
\hline 3 & Sleep mode & No & No & No & No & No & No \\
\hline 4 & $\begin{array}{l}\text { Unidirectional } \\
\text { link support }\end{array}$ & No & No & No & Yes & No & Yes \\
\hline 5 & Multicasting & Yes & Yes & Yes & No & Yes & No \\
\hline 6 & $\begin{array}{l}\text { On-demand } \\
\text { routing } \\
\text { behavior }\end{array}$ & Reactive & Reactive & Reactive & Reactive & Reactive & Reactive \\
\hline 7 & $\begin{array}{l}\text { Distributed } \\
\text { environment }\end{array}$ & Yes & Yes & Yes & Yes & Yes & Yes \\
\hline 8 & $\begin{array}{l}\text { Periodic } \\
\text { broadcast }\end{array}$ & Yes & Yes & Yes & Yes & Yes & Yes \\
\hline 9 & scalability & Yes & Yes & Yes & Yes & Yes & Yes \\
\hline 10 & $\begin{array}{l}\text { Routing } \\
\text { scheme }\end{array}$ & Flat & Flat & Flat & Flat & Flat & Flat \\
\hline 11 & Routing metric & $\begin{array}{l}\text { Associativity } \\
\text { between nodes }\end{array}$ & $\begin{array}{l}\text { Shortest } \\
\text { path }\end{array}$ & $\begin{array}{l}\text { Shortest } \\
\text { path }\end{array}$ & $\begin{array}{l}\text { Shortest } \\
\text { path }\end{array}$ & $\begin{array}{l}\text { Shortest } \\
\text { path }\end{array}$ & $\begin{array}{l}\text { Shortest } \\
\text { path }\end{array}$ \\
\hline 12 & $\begin{array}{l}\text { Route } \\
\text { optimization. }\end{array}$ & Yes & yes & Yes & yes & Yes & yes \\
\hline
\end{tabular}


6.1 Security:

Not all of the reactive protocols address the security vulnerabilities that are obvious in wireless networks. The proper function of these protocols is based on an assumption that all the nodes exist and operate in a secure environment where link- and physical-layer security mechanisms are in place. TORA and ABR are more secure than AODV, DSR. Although security is a major concern in wireless communications, it is found that the security mechanisms will increase processing time, power consumption, and latency. Note that reactive routing protocols already suffer from high latency in the network.

\subsection{Loop freedom:}

All tested reactive protocols are loop-free. No protocol addresses security vulnerabilities that exist in a wireless ad hoc network. However, there are certain proposals for providing secure routing at Layer 3 for all tested protocols.

\subsection{Sleep mode}

None of the reactive protocols supports the 'sleep mode', another important factor for power preservation, especially in battery-powered mobile nodes. AODV will consume more power than DSR owing to the exchange of periodic HELLO messages.

\subsection{Unidirectional link support}

Unidirectional links exist in wireless Communications and they should be supported in order to take advantage of any possible paths from a source node to a destination node. In MANETs, especially, there is no such 'luxury' as ignoring any possible paths, as routing protocols should take advantage of any link to calculate routes in the network. TORA designers take into account these limitations of the wireless network and support both bidirectional and unidirectional links. Only DSR in its current state, without any modification, can support both bidirectional and unidirectional links. However, DSR will introduce high routing overhead as routing information is stored at the data packets' header. Thus DSR will not scale well in large networks if communicating nodes are located at opposite edges of the network.

6.5 Multicasting: DSR and TORA do not provide multicasting. ABR, AODV, LAR provide multicasting. 6.6 On-demand routing behavior: All the reactive routing protocol shows on demand behavior.

6.7 Distributed environment: It indicates the way of interconnecting nodes under distributed environment and all the mentioned provide distributed environment.

6.8 Periodic broadcast: It represents sending packets periodically and it is seen that all the reactive protocols shows periodic broad cast mechanism.

6.9 Scalability: The up gradation of no. of nodes in the given environment is shown by all the reactive protocols.

6.10 Routing scheme: It indicates that all the routing protocols have flat routing mechanism.

6.11 Routing metric: It provides the path of connecting nodes for sending packets and it is seen that all he reactive routing protocols shows shortest path routing except ABR which is based on associativity between nodes.

6.12 Route optimization. The route has to be optimized for qualitative performance of sending packets .all the reactive routing protocol gives route optimization.

By summarizing the above results, it is seen that AODV, DSR, TORA are closer to the IETF MANET working group design recommendations. However, it is mainly up to the network designer to decide what he really needs from a network.

Given qualitative metrics and the attributes of the six protocols, it is suggested that AODV, DSR, TORA would be good candidates for the routing protocol in mobile ad hoc wireless networks. Therefore AODV, DSR, TORA are chosen for further evaluation in these simulations.

\section{Performance Evaluation Based On Quantitative Metrics}

The MANET network simulations are implemented using NS-2 simulator [23]. Nodes in the simulation move according to a model that we call Random Waypoint Mobility model [24]. Each node is then assigned a particular trajectory. The simulation period for each scenario is 500 seconds and the simulated mobility network area is $1000 \mathrm{~m}$ x $1000 \mathrm{~m}$ rectangle. In each simulation scenario, the nodes are initially located at the center of the simulation region. The nodes start moving after the first 10 seconds of simulated time. The MAC layer protocol IEEE 802.11 is used in all simulations with the data rate $11 \mathrm{Mbps}$. The transmission range is $500 \mathrm{~m}$. The application used to generate is CBR traffic and IP is used as Network layer protocol. 
The packet size 512 bytes. The node speeds is taken as $20 \mathrm{~m} / \mathrm{s}$. the no. of nodes are taken as 50 .The Performance evaluation, as well as the design and development of routing protocols for MANETs, requires additional parameters which is addressed in RFC developed by Internet Engineering Task Force (IETF).

There are two scenarios: 1) impact of varying node density, 2) impact of varying pause time.

\subsection{Experiment -1: Impact of varying node density}

In this set of the simulation, the number of nodes is varied in the network. The objective of this is to investigate the impact of node density on the protocol's performance. The same simulation area is used is $1000 \mathrm{~m} \times 1000 \mathrm{~m}$ and gradually increases the number of nodes in the network. A desirable property of a protocol is to have stable behavior regardless of the number of nodes in the network. A small number of nodes in a large simulation area will result in low connectivity due to the large distances between nodes. In contrast, a large number of nodes in a small simulation area will result in signal interference, as nodes are located very close to each other. As shown in fig 7.1(a) PDR of DSR is better than AODV and TORA. TORA shows least PDR for less no. of nodes, performance of TORA is better but as node increases PDR of TORA decreases.

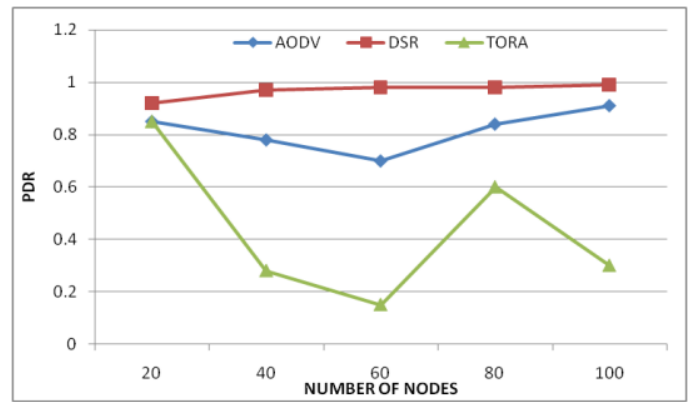

Fig 7.1 (a) PDR for varying node density

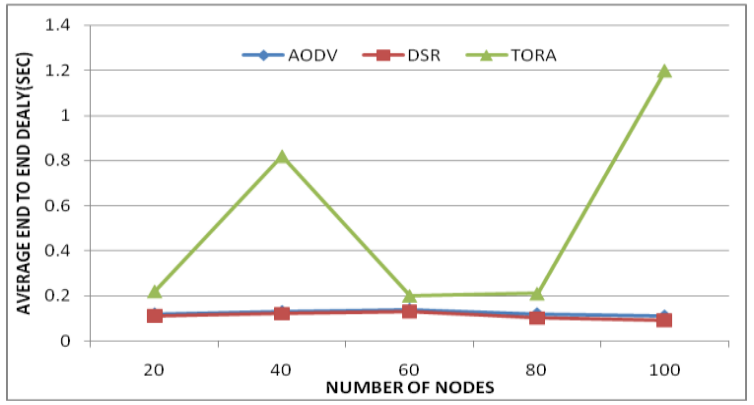

Fig.7.1 (b): Average end to end delay for varying node density

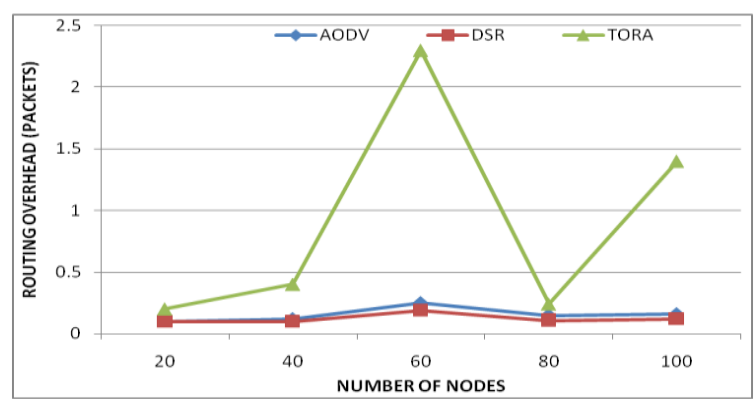

Fig.7.1 (c): Routing load for varying node density.

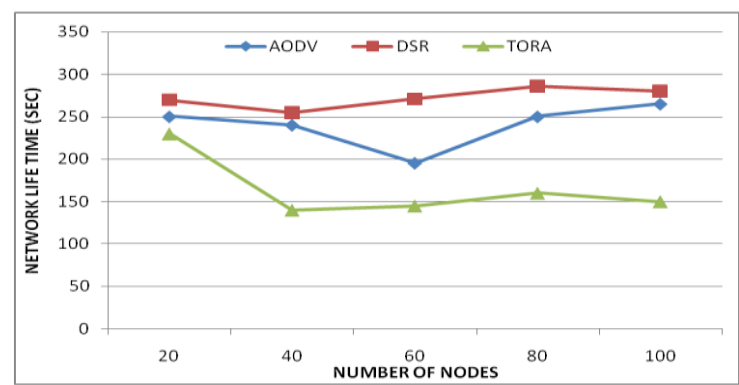

Fig.7.1 (d): Network life time for varying node density.

As shown in fig 7.1 (b) Average end to end delay, the performance of DSR and AODV are almost uniform. However, the performance of TORA is degrading due to increase in the number of nodes. In TORA, the end-to-end delay varies as the change in number of nodes. The direct result of a congested network is a high end-to-end delay for TORA, which increases exponentially in the case of 100 nodes. AODV and DSR present small fluctuations in terms of the end-to-end delay, but generally its performance is stable in all cases.

Figure 7.1 (c) shows the normalized routing load. TORA has a higher normalized routing load than AODV and DSR in the case of 40 and 80 nodes. However, AODV and DSR has similar routing overload when the number of nodes in the network increases.

Fig 7.1 (d) shows network life time which indicates that DSR has highest network life time and TORA has lowest network life time.

Thus from all these graphs it is clear that routing overhead increase with increase in number of nodes. DSR has better packet delivery ratio and in overall DSR has better performance. TORA has low performance because in our simulation network has middle dynamic. AODV and DSR have middle performance.

\subsection{Experiment -2: Impact of varying pause time}

As shown in fig 7.2 (a) DSR in this simulation experiment shows to have the best overall performance. On-demand protocols (DSR and AODV) drop a considerable number of packets during the route discovery phase, as route acquisition takes time proportional to the distance between the source and destination. Buffering of data packets while route discovery in progress, has a great potential of improving DSR, AODV and TORA performances. AODV has a slightly lower packet delivery performance than DSR because of higher drop rates. AODV uses route expiry, dropping some packets when a route expires and a new route must be found. 
Qualitative And Quantitative Performance Evaluation Of Adhoc On Demannd Routing Protocol In

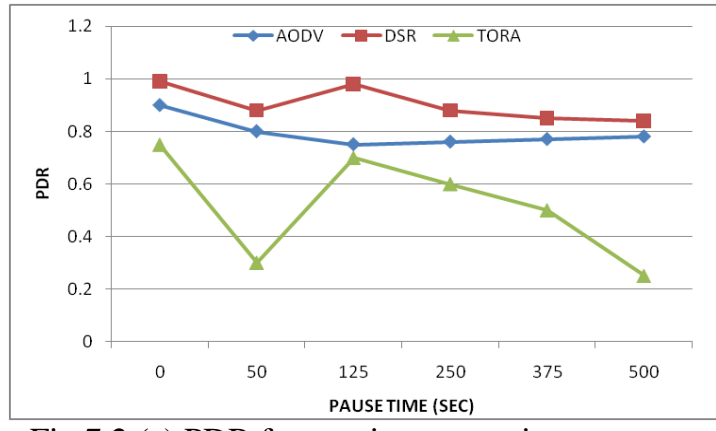

Fig 7.2 (a) PDR for varying pause time time

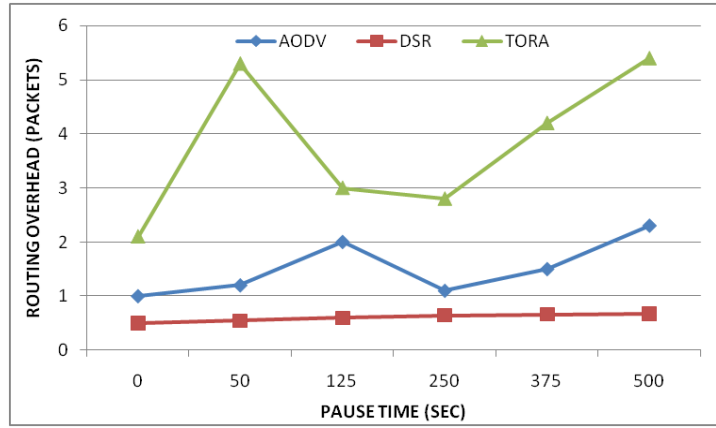

Fig 7.2 (c) Routing overhead for varying pause time time

As shown in fig 7.2 (b), it shows that the average end to end delay is similar for AODV and DSR and less than TORA.As shown in fig 7.2 (c), routing overload of TORA is higher than AODV and DSR.DSR has least routing overload. Network life time of DSR and AODV is higher than TORA as shown in fig 7.2 (d).

It is concluded from all the simulations results as follow. In case of Packet Delivery Ratio, DSR has better performance. In cases of Network Life Time, DSR and AODV have similar performance. In case of End-to-End Delay, AODV and DSR have similar performance. In case of Routing Overhead, DSR has better performance and TORA has worst performance.DSR has best performance for network life time.

\section{Conclusion}

The goal of this paper was performance evaluation/ comparison of a MANET between AODV, DSR and TORA routing protocols. In this paper, the performance of the six MANET reactive routing protocols was analyzed qualitatively and then using NS-2 simulations AODV, DSR and TORA was analyzed for qualitative metrics. It is observed that TORA had the lowest performance in terms of the packet delivery ratio in all of the simulations. DSR presented the lowest end-to-end delay in almost all of the simulations, and in most cases the end-to-end delay was independent of the varying simulation parameters. AODV is a good compromise when combining the protocol performance, in terms of the packet delivery ratio and the end-to-end delay. In addition, AODV performance in terms of end-to-end delay and network life time was very close to that of DSR. It was concluded that AODV was the appropriate protocol for any kind of application (voice, video, file transfer, etc.) in networks with high mobility that consist of up to 100 or more nodes. AODV in this simulation experiment shows to have the overall best performance. It has an improvement of DSR and TORA and has advantages of both of them. It often serves as the underlying protocol for lightweight adaptive multicast algorithms, whereas DSR suits for network in which mobiles move at moderate speed. It has a significant overhead as the packet size is large carrying full routing information.

\section{References}

[1] C.Sivaram murthy, B.S.Manoj, “Adhoc wireless networks:Architectures, and protocols”, Pearson Education, 2004.

[2] T. Clausen and P. Jacquet (2003), "Optimized Link State Routing Protocol (OLSR)", RFC 3262, IETF Network Working Group, October 2003

[3] C. Perkins, E. B. Royer and S. Das (2003), "Ad hoc On-Demand Distance Vector (AODV) Routing", RFC 3561, IETF Network Working Group, July.

[4] Mohammed Bouhorma, H.Bentaouit and A.Boudhir, "Performance comparison of Ad hoc Routing protocols AODV and DSR" ,IEEE 2009.

[5] R. Misra, C. R. Manda (2005)1, "Performance Comparison of AODV/DSR On-Demand Routing Protocols for Ad Hoc Networks in Constrained Situation", IEEE ICPWC 2005 
[6]. Ahmed S, Alam MS. "Performance evaluation of important ad hoc network protocols." EURASIP Journal on Wireless Communications and Networking 2006; (2): 42-42.

[7] S. R. Das, C. E. Perkins and E. M. Royer (2000), "Performance comparison of Two On-Demand Routing protocols for Ad hoc Networks", In Proc. of INFOC OM 2000, Tel Aviv, Israel, March 2000.

[8] Kumar BRA, Reddy LC, Hiremath PS." Performance comparison of wireless mobile ad-hoc network routing protocols." International Journal of Computer Science and Network Security 2008; 8(6): 337-343.

[9]. Rahman AHA, Zukarnain ZA." Performance comparison of AODV, DSDV and I-DSDV routing protocols in mobile ad hoc networks.”, European Journal of Scientific Research 2009; 31(4): 566-576.

[10]. Divecha A, Grosan AC, Sanya S." Impact of node mobility on MANET routing protocols models", Journal of Digital Information Management 2007; 5: 19-23.

[11] H. Ehsan and Z. A. Uzmi (2004), "Performance Comparison of Ad HocWireless Network Routing Protocols", IEEE INMIC 2004.

[12] F. Bertocchi, P. Bergamo, G. Mazzin (2003), "Performance Comparison of Routing Protocols for Ad hoc Networks", IEEE GLOBECOM 2003

[13] Mehran Abolhasan, Tadeusz Wysocki and Eryk Dutkiewicz," A review of routing protocols for mobile ad hoc networks", Elsevier 2003.

[14] Clausen T, Jacquet P (eds). “Optimized Link State Routing Protocol (OLSR).” RFC 3626, 2003.

[15] Perkins CE, Bhagwat P.” highly dynamic destination-sequenced distance-vector routing (DSDV) for mobile computers" Computer Communications Review 1994; 234-244.

[16] Das SR, Perkins CE, Royer EM.” Performance comparison of two on-demand routing protocols for ad hoc networks.” Nineteenth Annual Joint Conference of the IEEE Computer and Communications Societies. Proceedings of the IEEE 2000; 1: 3-12.

[17] D.Johnson, "The Dynamic Source Routing Protocol (DSR)", RFC4728, Feb 2007.

[18] Yih-Chun Hu and David B.Johnson ",Caching Strategies in On-Demand Routing Protocols for Wireless Ad hoc Networks",ACM 2000

[19] V. Nazari, K. Ziarati (2006), "Performance Comparison of Routing Protocols for Mobile Ad hoc Networks", IEEE 2006.

[20] Haas ZJ, Pearlman MR, Samar P. The Zone Routing Protocol (ZRP) for ad hoc networks. Draft: draft-ietf-manet-zone-zrp-04.txt, 2002.

[21] Carp B, Kung HT. GPSR: "greedy perimeter stateless routing for wireless networks." In Proceedings of the Sixth Annual ACM/IEEE International Conference on Mobile Computing and Networking (MobiCom 2000).

[22] Corson S, Macker J (eds). "Mobile Ad hoc Networking (MANET): routing protocol performance issues and evaluation considerations". RFC 2501, 1999.

[23] "The network simulator ns-2. http://www.isi.edu/nsnam/ns2,"

[24] Tracy Camp, Jeff Boleng and Vanessa Davies (2002), "A survey of Mobility Models for Ad hoc Network Research", Wireless Communications and Mobile computing: A special issue on Ad hoc network Research, vol 2, No5, pp. 483-502, 2002.

[25] Chakeres, I. Perkins, C. "Dynamic MANET On-Demand (DYMO) Routing", IETF Internet-Draft, draft-ietfmanetdymo-17.txt. 2010.

[26] V. Kanakaris*, D. Ndzi and D. Azzi., Ad-hoc Networks Energy Consumption: A review of the Adhoc Routing Protocols, Journal of Engineering Science and Technology Review 3 (1) (July 2010).

[28] Y.-B. Ko, N.H. Vaidya, "Location-aided routing (LAR) in mobile ad hoc networks", in: Proceedings of the Fourth Annual ACM/IEEE International Conference on Mobile Computing and Networking (Mobicom_98), Dallas, TX,1998.

[28] P. Sinha, R.Sivakumar and V. Bharghaven, “CEDAR: a Core-Extraction Distributed Ad hoc Routing algorithm” IEEE INFOCOM, March 1999.

[29] M. Kummakasikit, S.Thipchaksurat " Performance Improvement of Associativity-Based Routing for Ad-Hoc Mobile Networks" presented in International conference of Information, Communications and Signal Processing, 2006.

\section{AUTHORS}

First Author - Patil V.P, Smt. Indira Gandhi College of Engineering, New Mumbai, India,

Er. PATIL V.P. is currently working as a faculty member in Electronics and Telecommunication Engineering department in Smt. Indira Gandhi college of Engineering, New Mumbai. He is graduate in B.E. and post graduate in M.TECH (ELECTRONICS DESIGN AND TECHNOLOGY) . He is having 25 years of experience in teaching in engineering colleges. His area of research is in computer communication networking and microwave engineering. 